\title{
A QUMOND galactic $N$-body code - I. Poisson solver and rotation curve fitting
}

\author{
G. W. Angus, ${ }^{1 \star}$ K. J. van der Heyden, ${ }^{1}$ B. Famaey, ${ }^{2,3}$ G. Gentile, ${ }^{4}$ S. S. McGaugh ${ }^{5}$ \\ and W. J. G. de Blok ${ }^{1}$ \\ ${ }^{1}$ Astrophysics, Cosmology \& Gravity Centre, University of Cape Town, Private Bag X3, Rondebosch 7701, South Africa \\ ${ }^{2}$ Observatoire Astronomique de Strasbourg, CNRS, UMR 7550, France \\ ${ }^{3}$ AIfA, University of Bonn, Auf dem Hügel 71, D-53121 Bonn, Germany \\ ${ }^{4}$ Sterrenkundig Observatorium, Ghent University, Krijgslaan 281, S9, 9000 Ghent, Belgium \\ ${ }^{5}$ Department of Astronomy, Computer \& Space Sciences Bldg, University of Maryland, College Park, MD 20742-2421, USA
}

Accepted 2012 January 3. Received 2011 December 28; in original form 2011 July 1

\begin{abstract}
Here we present a new particle-mesh galactic $N$-body code that uses the full multigrid algorithm for solving the modified Poisson equation of the quasi-linear formulation of modified Newtonian dynamics (QUMOND). A novel approach for handling the boundary conditions using a refinement strategy is implemented and the accuracy of the code is compared with analytical solutions of Kuzmin discs. We then employ the code to compute the predicted rotation curves for a sample of five spiral galaxies from the THINGS sample. We generated static $\mathrm{N}$-body realizations of the galaxies according to their stellar and gaseous surface densities and allowed their distances, mass-to-light ratios ( $M / L$ values) and both the stellar and gas scaleheights to vary in order to estimate the best-fitting parameters. We found that NGC 3621, NGC 3521 and DDO 154 are well fitted by MOND using expected values of the distance and $M / L$. NGC 2403 required a moderately larger $M / L$ than expected and NGC 2903 required a substantially larger value. The surprising result was that the scale-height of the dominant baryonic component was well constrained by the rotation curves: the gas scale-height for DDO 154 and the stellar scale-height for the others. In fact, if the suggested stellar scale-height (one-fifth the stellar scale-length) was used in the case of NGC 3621 and NGC 3521 it would not be possible to produce a good fit to the inner rotation curve. For each of the four stellar dominated galaxies, we calculated the vertical velocity dispersions which we found to be, on the whole, quite typical compared with observed stellar vertical velocity dispersions of face-on spirals. We conclude that modelling the gas scale-heights of the gas-rich dwarf spiral galaxies will be vital in order to make precise conclusions about MOND.
\end{abstract}

Key words: galaxies: kinematics and dynamics - galaxies: photometry - galaxies: spiral cosmology: dark matter.

\section{INTRODUCTION}

The Hi Nearby Galaxy Survey (THINGS; Walter et al. 2008) brought an unprecedented level of precision to the measurement of the rotation curves of certain nearby spiral galaxies. This, when coupled with $3.6 \mu \mathrm{m}$ images of the stellar component from the Spitzer Infrared Nearby Galaxies Survey (SINGS; Kennicutt et al. 2003), produces a stringent new data set for modelling the galactic dynamics of these systems. These tighter constraints are of paramount importance for testing alternative theories of gravity, in particular those with no galactic dark matter like modified Newtonian

^E-mail: angus.gz@gmail.com dynamics (MOND; Milgrom 1983, but see also Famaey \& McGaugh 2011).

MOND is an appealing framework because, at least in galaxies, the gravitational field is fully determined by the matter distribution of the visible components. This means that a galaxy comprising discy stellar and gaseous components produces a rotation curve depending only on the properties of those components. It is crucial, in order to keep up with the advancing observations, that we produce methods of modelling the galaxies with a similar level of sophistication.

In this paper, we develop a galactic $N$-body code for MOND and apply it to fitting the high-resolution rotation curves of the THINGS survey. We compare predicted and fitted distances and make use of 
free parameters in the form of the scale-heights of the two baryonic components.

\section{SOLVING THE MOND EQUATION ON A GRID}

MOND has a different force law from Newtonian dynamics, and this traditionally is rather tricky to solve (Bekenstein \& Milgrom 1984; Milgrom 1986; Brada \& Milgrom 1995). For instance, in the Newtonian analogue, the ordinary Poisson equation must simply be solved using the matter distribution in terms of its density, which includes stars $\left(\rho_{*}\right)$, gas $\left(\rho_{\mathrm{g}}\right)$ and cold dark matter $\left(\rho_{\mathrm{CDM}}\right)$, using

$\nabla^{2} \Phi_{\mathrm{N}}=4 \pi G\left(\rho_{*}+\rho_{\mathrm{g}}+\rho_{\mathrm{CDM}}\right)$

to give the Newtonian potential, $\Phi_{\mathrm{N}}$. However, in a recently proposed version of MOND, called QUMOND (Milgrom 2010; but see also Zhao \& Famaey 2010), it was shown that the MOND potential, $\Phi$, can be found exactly from the Newtonian potential (not including cold dark matter) as follows:

$\nabla^{2} \Phi=\nabla \cdot\left[v(y) \nabla \Phi_{\mathrm{N}}\right]$,

where $v(y)=0.5+0.5 \sqrt{1+4 / y}$ and $y=\nabla \Phi_{\mathrm{N}} / a_{0}$, with $a_{0}$ being the MOND acceleration constant chosen here to be $3.6\left(\mathrm{~km} \mathrm{~s}^{-1}\right)^{2} \mathrm{pc}^{-1} . v(y)$ could take on another form, and similarly $a_{0}$ could take on a different value, but we choose here not to focus on this topic for fear of being sidetracked. We note that the $v$-function we adopt here is the analogue of the $\mu$-function used by Famaey \& Binney (2005) to fit the terminal velocity curve of the Milky Way.

Very often in the literature, equation (2) is not solved, but rather an approximation is made that is referred to as the following algebraic MOND relation:

$\nabla \Phi=v(y) \nabla \Phi_{\mathrm{N}}$.

This approximation ignores the curl field, which is negligible in most cases of high symmetry, but has the scope to be a significant component of the gravitational field in triaxial systems. Here we are not advocating that it is imperative to use equation (2) for fitting rotation curves of relaxed spiral galaxies, but rather for when MOND is extended to study interacting galaxies, or galaxies with lopsided matter distributions.

Our goal is to determine the gravity at locations inside the galaxy we are studying and the procedure to do this is as follows. We must set up a three-dimensional grid, sliced into numerous cubic cells usually we use 65,129 or 257 cells per dimension. In each cell we have the following:

(i) an approximated, but near exact, value of the baryonic density (step 1, the source of equation 1 using only gas and stars);

(ii) a solution to the Newtonian potential (step 2);

(iii) the source 'density' - the right-hand side of equation (2) (step 3);

(iv) the solution of the QUMOND potential (step 4).

Getting from step 1 to step 4 is in the details of the code, which we briefly describe here.

We read in a set of particle positions that determine the density of the stars and gas in the galaxy on the grid using the cloud-incell technique (step 1). The stars and gas are allocated half of the total number of particles each and therefore the particles of gas and stars are weighted differently and according to the total mass of each component. With this density, we solve for the Newtonian potential (step 2; equation 1) using the full multigrid algorithm that is well known and described in Press et al. (1992). The full multigrid method is extended to three dimensions which implements redblack Gauss-Seidel relaxation. Our default is to use six V-cycles, from the finest to the coarsest grid and back to the finest with bilinear prolongation and restriction, as well as two pre- and post-smoothing steps before and after the coarse grid correction is computed.

We then use finite differencing to find the source for equation (2) (step 3). The finite differencing technique used to solve equation (2) is described in Angus \& Diaferio (2011). We use the same multigrid technique to solve equations (1) and (2), only the source changes (step 4). We make a final finite differencing to determine the QUMOND gravity, $\nabla \Phi$, on the grid and then we interpolate to the point at which we wish to know the gravity. The interpolation scheme is the same as the scheme to assign density to the grid, i.e. the cloud-in-cell technique.

Our final step is to calculate the circular speed associated with this QUMOND potential as a function of radius. This is taken as

$v_{\mathrm{c}}^{2}=R \times\left.\partial_{R} \Phi\right|_{z=0}$.

Although this is the first attempt to solve the modified Poisson equation of QUMOND on a grid in a non-cosmological setting (see Llinares, Knebe \& Zhao 2008; Angus \& Diaferio 2011; Llinares 2011 for the cosmological analogue), this is not the first time that a modified Poisson equation linked to MOND has been solved in such a way. Brada \& Milgrom (1999) developed a cubic particlemesh code that solved the modified Poisson equation and used it to investigate some important topics (Brada \& Milgrom 2000a,b). Tiret \& Combes (2007) developed a similar code and parallelized it and incorporated hydrodynamics into their simulations (see Tiret \& Combes 2008a,b). In addition to these, Londrillo \& Nipoti (2009) produced a spherical grid code that investigated various issues in the framework of MOND (Nipoti, Londrillo \& Ciotti 2007a,b; Nipoti et al. 2008).

The key to a galactic $N$-body code, as opposed to a cosmological one, is the boundary conditions. In a cosmological code, the expectation that the Universe is homogeneous and isotropic allows us to enforce periodic boundary conditions. At the galaxy scale, the boundary conditions must be set precisely at all outer grid cells, which is non-trivial in MOND. It is not clear how accurately this was achieved in the previous codes, but here we implement a different strategy (see Tiret \& Combes 2007; Wu et al. 2009 for other examples).

We define a coarse grid which is many times $\left(2^{10}\right)$ larger than the galaxy we are studying. For this coarsest grid we set the boundary condition of the Newtonian and QUMOND potentials to be zero. Then we solve for the Newtonian and QUMOND potentials everywhere on the grid. From this, we define a refined grid that is half the size of the coarse grid and we interpolate through the values of the potential on the coarse grid to define the boundary condition of the refined grid. We make this refinement up to 10 times in order to zoom in on our galaxy, but the boundary conditions are correct at the sub-per cent level at all points on the grid by the second refinement. This also enables us to use different grid levels to find the potential at the location of a particle, depending on its position in the various grid levels. The limitation of this is that it is centrally refining, not arbitrarily towards regions of high density. Note that the number of grid cells is constant for each refinement, so the resolution increases for the finer grids - with more cells per kpc.

There is an initial guess that is required in multigrid methods. For clarity, our finest grid is $257 \times 257 \times 257$ and we solve for the potential on this grid at 10 incremental levels of resolution (box size). For each box size, in order to more rapidly calculate the potential on the finest grid, we smooth the calculated (fed in) 
density down to the coarsest possible level of resolution, which is a $3 \times 3 \times 3$ grid. The coarsest density field is known in each of the 27 cells and we must solve for the potential in each of these cells. Since for the largest box, of length $4 \mathrm{Mpc}$, the density and potential in each of the 26 outer cells are zero, the potential in the central cell is simply proportional to the density in that central cell. This is true for both the Newtonian step and the QUMOND step. Following this, we perform the standard multigrid interpolation of the potential to finer grids and use the finer density defined on those grids to make a more rapid calculation of the potential on the finest grid.

\section{STATIC $N$-BODY REALIZATIONS}

Our aim is to use this particle-mesh code to fit rotation curves. To do this, we need to generate static $N$-body realizations of galaxies depending on their surface density. Initially, to test the resolution of the code, we generated a realization of a Kuzmin disc and compared the analytical circular velocity to the circular velocity simulated by the code.

\subsection{Kuzmin dises}

Kuzmin discs are unique in MOND because their isopotential contours are spherically symmetric (at least in each distinct hemisphere, above and below the disc mid-plane), meaning that there is no curl field. The surface density of a Kuzmin disc is given by Binney \& Tremaine (2008) to be $\Sigma_{\mathrm{K}}(R)=\frac{a M}{2 \pi}\left(R^{2}+a^{2}\right)^{-3 / 2}$. Assuming an infinitely thin disc, and integrating this by $2 \pi R \mathrm{~d} R$, we get the enclosed mass $M_{\mathrm{K}}(R)=M\left(1-\frac{a}{\sqrt{R^{2}+a^{2}}}\right)$. If we express the fraction of the total mass as $f=M_{\mathrm{K}}(R) / M$, then we know that the way to distribute particles in radius is $R=a \sqrt{(1-f)^{-2}-1}$. From this we randomly sample $f$ and distribute the particles according to a random azimuthal angle.

We did this for a model where we set $a=1.5 \mathrm{kpc}$ and $M=$ $10^{10} \mathrm{M}_{\odot}$ and we show in Fig. 1 (left-hand panel) the fractional difference between this, the simulated QUMOND rotation curve and the analytically expected rotation curve. For the Kuzmin disc, the three-dimensional Newtonian gravity on a star slightly below the disc is defined by $g_{\mathrm{N}, r}=-G M /\left(R^{2}+a^{2}\right)$ and this points towards the position $(R, z)=(0, a)$, as if all the mass were concen- trated in a single point at the location $(0, a)$. Similarly, the gravity towards the centre of the disc is $g_{\mathrm{N}, R}=-G M R\left(R^{2}+a^{2}\right)^{-3 / 2}$. Therefore, the QUMOND gravity towards the centre of the disc is $g_{M, R}=v\left(\left|g_{\mathrm{N}, r}\right| / a_{0}\right) g_{\mathrm{N}, R}$. Note the subtlety here with respect to the subscripts of $R$ and $r$.

Fig. 1 demonstrates that the accuracy of the code is less than 1 per cent beyond $0.3 \mathrm{kpc}$ and less than 0.1 per cent beyond $1 \mathrm{kpc}$ for this model. This depends greatly on the extent of the galaxy. A Kuzmin disc, with scale radius $a=1.5 \mathrm{kpc}$ still has 5 per cent of its mass beyond $30 \mathrm{kpc}$ and so is rather unrealistically extended. In Fig. 1 (right-hand panel) we make the same plots for $a=150 \mathrm{pc}$ and $M=10^{9} \mathrm{M}_{\odot}$ to demonstrate that in that case the agreement is better than 0.1 per cent down to $\sim 50 \mathrm{pc}$. In both cases, as for the real galaxies to follow, we use the following strategy to evaluate gravity at various radii, $R$. When $R<0.8 \mathrm{kpc}$ we use the finest grid which has a default box size of $4 \mathrm{kpc}$, when $0.8<R<1.6 \mathrm{kpc}$ we use the second finest box of size $8 \mathrm{kpc}$, when $1.6<R<3.2 \mathrm{kpc}$ we use the 16-kpc box and so on. These values need not be fixed and should be tailored to different galaxy sizes.

\subsection{Convergence}

Although we can accurately simulate the gravitational field of a Kuzmin disc to roughly 0.1 per cent, it is equally important to ensure that the solution to Poisson's equation does converge for more complex density distributions, which might not immediately follow from a smooth Kuzmin disc. As mentioned above, our default parameters for using the full multigrid algorithm are that we make a standard number of six V-cycles at each level of the grid. Before and after we make the coarse grid correction we always make two red-black Gauss-Seidel relaxation sweeps.

For one of our galaxies, NGC 3621, we have plotted in Fig. 2 the relative error (as a function of radius) between the simulated rotation speed for a series of numbers of V-cycles compared to the rotation speed found using $99 \mathrm{~V}$-cycles. Each different number of $\mathrm{V}$-cycles is given a distinct colour. If a colour is not seen at a specific radius, this is because the relative error is less than $10^{-8}$ and is most likely identical to the $99 \mathrm{~V}$-cycles simulation. The discontinuities are caused by moving from one grid resolution to another. Clearly, using only one or two V-cycles does not allow for convergence, but by six cycles the difference is less than 0.1 per cent at all radii. Since the theoretical accuracy of the code is roughly 0.1 per cent as well
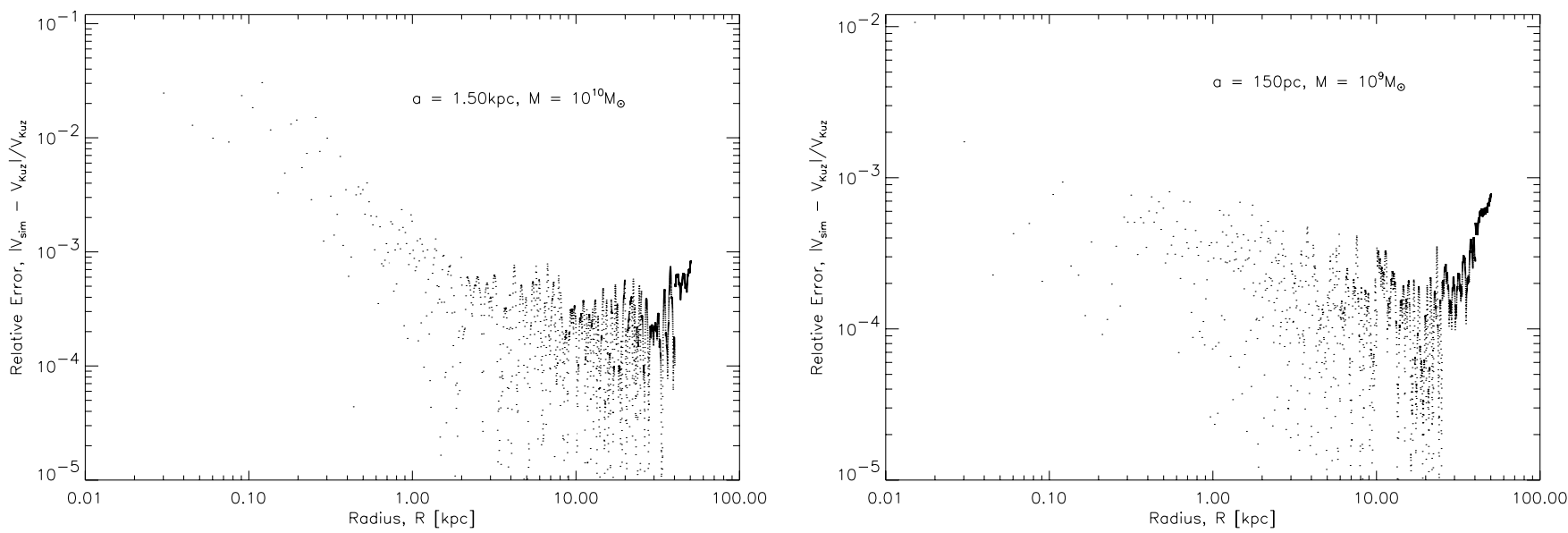

Figure 1. The relative error between the analytically defined QUMOND circular velocity and the simulated circular velocity of a Kuzmin disc. The left-hand panel uses a mass of $10^{10} \mathrm{M}_{\odot}$ and scale-length of $1.5 \mathrm{kpc}$, whereas the right-hand panel uses a mass of $10^{9} \mathrm{M}_{\odot}$ and scale-length of $150 \mathrm{pc}$. Note that the $y$-axes have different ranges. 


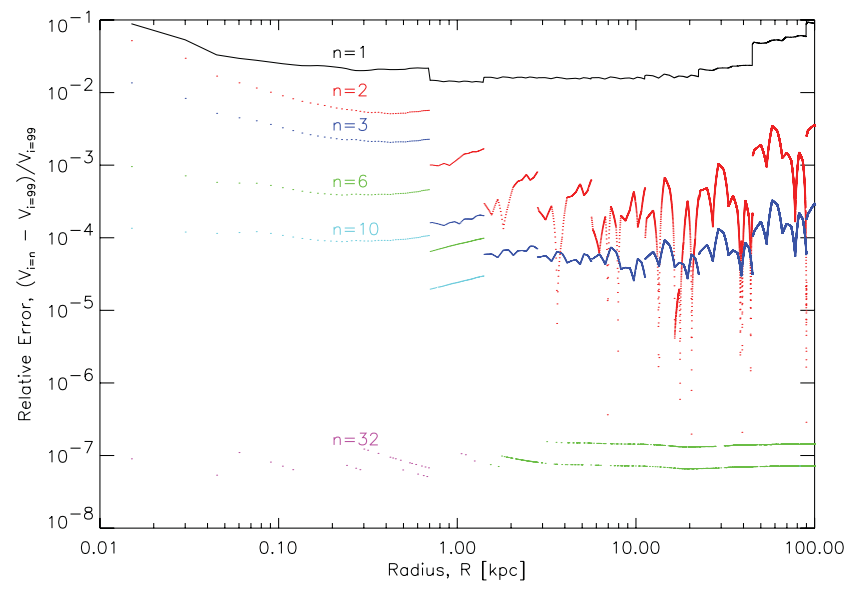

Figure 2. For NGC 3621, the relative error (as a function of radius) between the simulated rotation speed for a series of numbers of V-cycles compared to the rotation speed found using $99 \mathrm{~V}$-cycles. Each different number of $\mathrm{V}$-cycles is given a distinct colour. If a colour is not seen at a specific radius, this is because the relative error is less than $10^{-8}$. The discontinuities are caused by moving from one grid resolution to another.

(see Fig. 1), it makes six V-cycles a sensible number of cycles to make until the accuracy can be improved.

\subsection{Curl field and scale-height}

To compare the difference between solving the modified Poisson equation of QUMOND (equation 2) and the algebraic MOND relation (equation 3) we plot in Fig. 3 the rotation curve for an exponential disc galaxy with scale-length $a=1.5 \mathrm{kpc}$ and total mass $M=10^{10} \mathrm{M}_{\odot}$. There is a small but noticeable difference even for a perfectly symmetric and thin exponential disc. Also plotted is the simulated rotation curve for a disc with scale-height of $1 \mathrm{kpc}$, with a sech ${ }^{2}$ distribution. This creates a $10 \mathrm{~km} \mathrm{~s}^{-1}$ difference out to $R=$ $6 \mathrm{kpc}$ and is still significant at $R=10 \mathrm{kpc}$. For this reason, scaleheight is a crucial parameter that we will use to enhance the fits to the rotation curves of the galaxies.

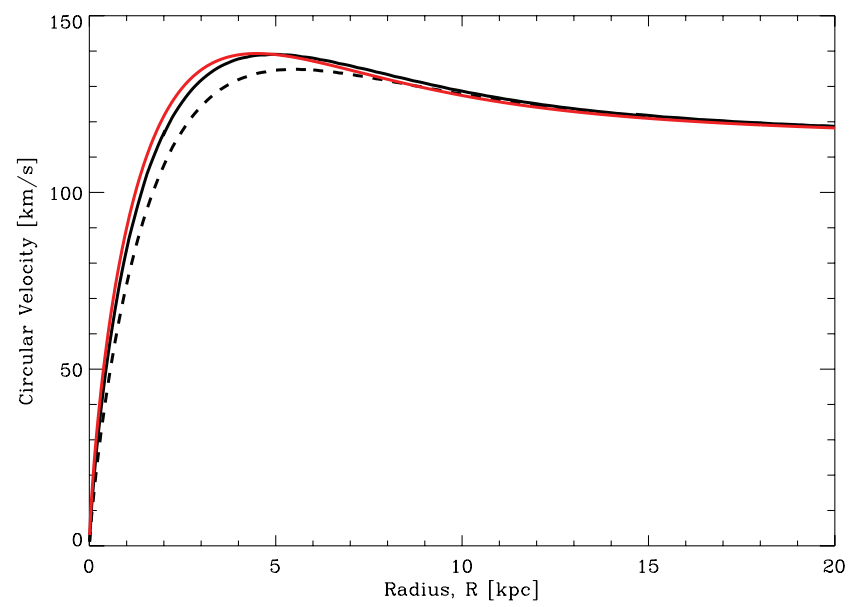

Figure 3. The difference between the rotation curve when we solve the modified Poisson equation of QUMOND (solid black, equation 2) and the algebraic relation (solid red, equation 3) for a razor thin exponential disc. The dashed curve shows the rotation speed from the same exponential disc, using our code, but with a vertical scale-height of $1 \mathrm{kpc}$ that is described by a sech ${ }^{2}$ distribution.

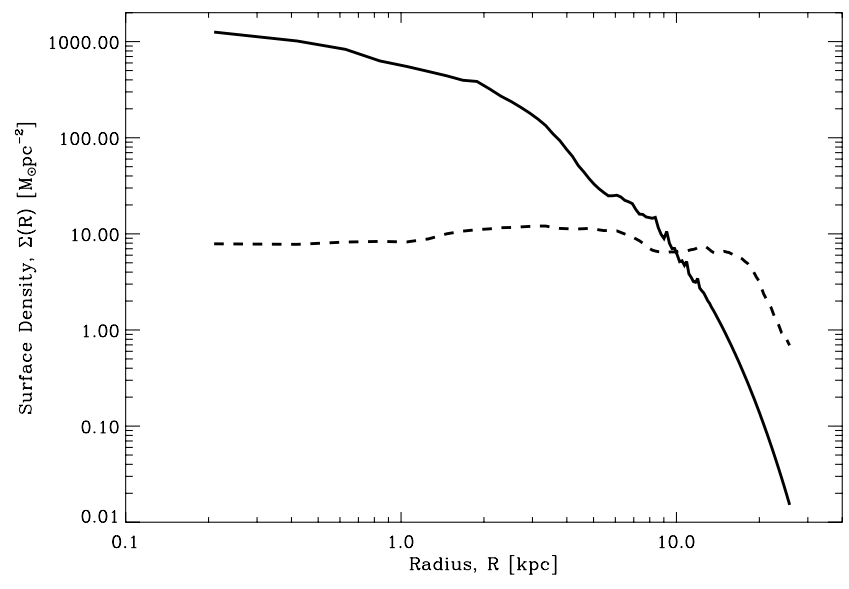

Figure 4. The surface densities of the stellar (solid) and gaseous (dashed) components for NGC 3621. Data comes from de Blok et al. (2008).

\subsection{Realistic two-component galaxies}

In order to generate realistic, static $N$-body realizations for galaxies we need the surface densities of both the stellar and gaseous discs, like that plotted for NGC 3621 in Fig. 4. In our models we always separate stars and gas, giving half of the particles to each component and weighting them according to the relative masses of these two components. We use the well-known rejection technique from Press et al. (1992) to produce an $N$-body realization of the two components that resembles the observed surface densities. Typically we use $256^{3}$ particles and our most refined grid is only $4 \mathrm{kpc}$ across, with 129 cells per dimension.

\subsection{Free parameters}

We are now in a position to compare simulated circular velocity curves with the observed rotation curves. This brings into question the various free parameters we employ. We assume that the uncertainty in the inclination of the galaxy is contained within the measurement errors associated with each data point. The error from inclination is addressed in de Blok et al. (2008), where the tilted ring fits find little variation in inclination for the galaxies we use. This leaves us with four free parameters: the distance to the galaxy, $D$, the mass-to-light ratio $(M / L)$ of the stellar component of the galaxy and the scale-heights of both the stellar, $z_{*}$, and gas, $z_{\mathrm{g}}$, discs - with $\operatorname{sech}^{2}$ distributions. We then make an exhaustive search through parameter space to find the quality of fit values, $\chi^{2} / n=\left\{\sum_{i=1}^{n} \frac{\left[V_{\text {fit }}\left(R_{i}\right)-V_{\text {obs }}\left(R_{i}\right)\right]^{2}}{\sigma_{\text {obs }}^{2}\left(R_{i}\right)}\right\} / n$, of these parameters by comparing the simulated QUMOND rotation curve with the observed one. We initially enforce flat priors on the distance to be no more than two standard deviations and the $M / L$ is forced to be within the range set by the diet-Salpeter and Kroupa initial mass functions (IMFs). We then relax these priors if no good fit is found, or if a significantly improved fit is found despite not being preferred by current limits on the parameters. The scale-heights are free to vary from razor thin up to $1 \mathrm{kpc}$. It should be emphasized that Gentile, Famaey \& de Blok (2011) have suggested that de Blok et al. (2008) overestimate the error bars on some rotation curves, $\sigma_{\mathrm{obs}}$, and as such the reduced $\chi^{2}$ cannot be directly used as a probability indicator in every case.

\section{SAMPLE}

The sample of five galaxies that we have chosen is a subsample of the galaxies used in the MOND fits of Gentile et al. (2011), which 

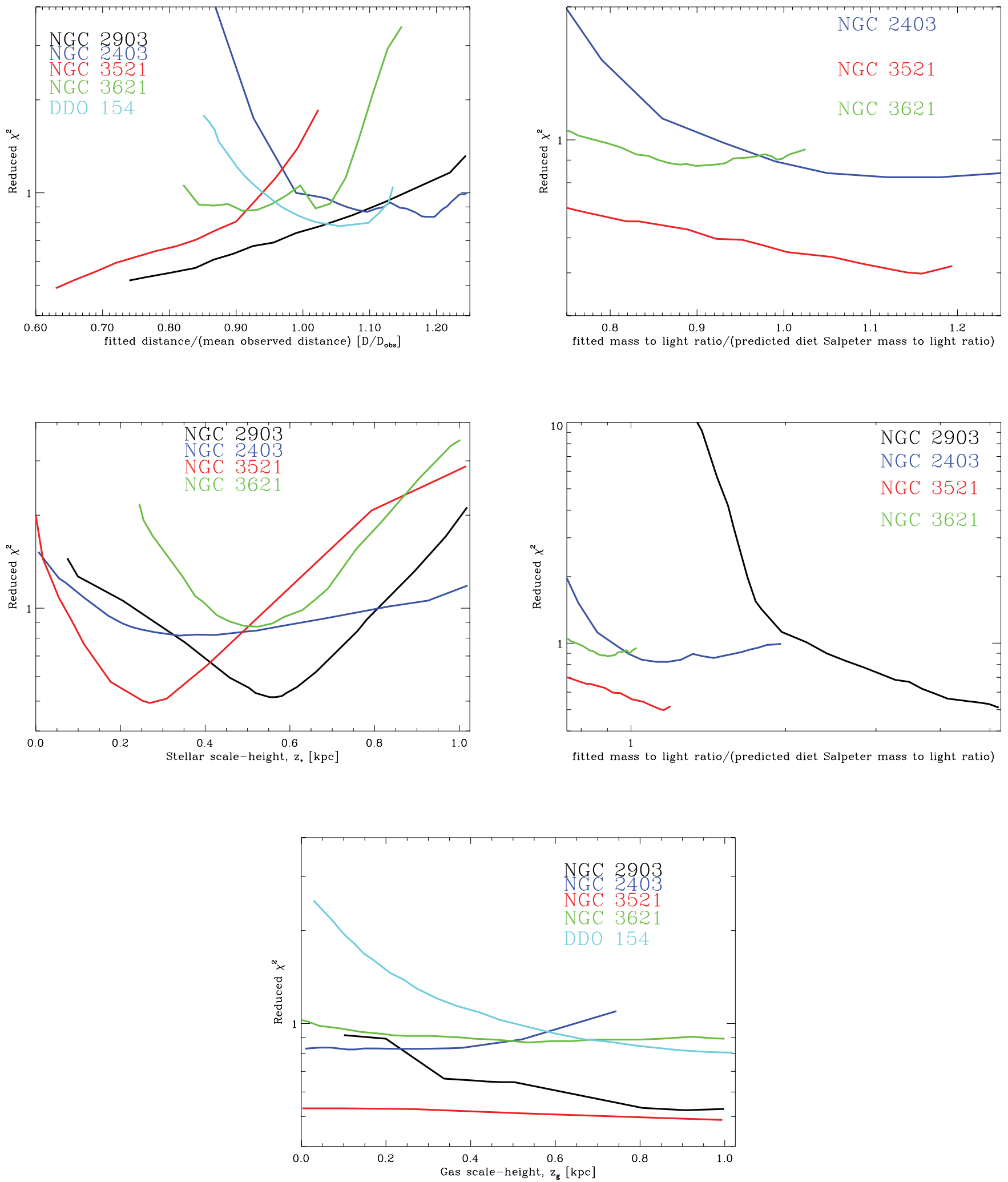

Figure 5. The curves of minimum reduced $\chi^{2}$ as a function of the fitted parameters of each galaxy. The top left-hand panel shows the fitted distance of each galaxy normalized to the observed distance, and the top right-hand panel shows the fitted $M / L$ normalized to the $M / L$ using the diet-Salpeter IMF (the figure below this is the same as above but with a larger plotted range). We also plot the two scale-heights. A different coloured line is used for each galaxy and a legend emphasizes this in each panel. 
is itself a subset of the galaxies studied by de Blok et al. (2008). Our sample removed all galaxies that had a significant spheroid component and also those galaxies that could be aided by the external field effect, which we devote a future paper to. Here our goal is not to have a representative sample of galaxies to test MOND in a statistical sense, but rather to highlight the potential of this Poisson solver and to investigate the impact of variable scale-heights on rotation curve fits. The properties of the five galaxies themselves can be found in detail in de Blok et al. (2008).

\section{RESULTS}

In Fig. 5 we plot the minimum reduced $\chi^{2}$ for each free parameter as a function of that parameter. Specifically, this is the minimum $\chi^{2}$ achievable with that parameter fixed and every other parameter free. The distances and $M / L$ are limited according to the plotted range for each galaxy.

Each galaxy in Fig. 5 has a different line colour. In the top lefthand panel, we plot reduced $\chi^{2}$ for each galaxy against distance. The fitted distance is normalized to the measured distance and similarly the fitted $M / L$ is normalized to the $M / L$ predicted by the diet-Salpeter IMF. The $1 \sigma$ error on distance varies from galaxy to galaxy and can be found in Table 1 . For $M / L$, the Kroupa IMF is typically 70 per cent of the diet-Salpeter value.

Clearly two galaxies (NGC 2903 and NGC 3521) prefer substantially lower distances than the measurements suggest, whereas the other three galaxies have their minima closer to the measured distance. It is worth bearing in mind that NGC 2903 and NGC 3521 have the largest uncertainties on their distances, 25 and 30 per cent, respectively.

The $M / L$ plots (top right and middle right) have only four lines since we fix the $M / L$ of DDO 154 to the diet-Salpeter value and do not vary it, nor do we vary its stellar scale-height. The concern here is that the curve for NGC 2903 rises sharply towards large $\chi^{2}$ when typical $M / L$ are tried.

The gas scale-height is only significant for two galaxies. Obviously, DDO 154 is our only gas-dominated galaxy and 95 per cent of its mass is gaseous. It prefers as large a gas scale-height as possible and the $\chi^{2}$ rises sharply below $0.6 \mathrm{kpc}$. On the other hand, NGC 2403 prefers a thin gas disc below $0.4 \mathrm{kpc}$ in scale-height. The other three galaxies prefer gas scale-heights larger than $0.5 \mathrm{kpc}$, but no significant gain is achieved for larger values than this.

The stellar scale-height is strongly constrained by the rotation curves. All four stellar dominated galaxies show a clear minimum between 0.25 and $0.55 \mathrm{kpc}$ and the quality of the fits would be significantly reduced if they were forced to be razor thin or $1 \mathrm{kpc}$ in extent.

In Figs 6-10 we plot various fits to the five rotation curves as well as three contour plots (DDO 154 only has one) for the three combinations of the three free parameters (gas scale-height has been omitted).

\subsection{DDO 154}

A lower $\chi^{2}$ can be achieved by increasing the distance and gas scale-height. In Table 1 we give the parameters used for the three fits in the left-hand panel of Fig. 6. In all figures showing the rotation curve, the fits and data points are rescaled to the distance of model (a) for that particular galaxy. In the case of DDO 154 this is 1.085 times the measured distance of $4.3 \mathrm{Mpc}$ and as such is $4.67 \mathrm{Mpc}$. It is not possible to fit the inner curve and outer flat data points beyond $7 \mathrm{kpc}$ with the same model. The flat outer points may be due to a warp in the outer part of its gaseous disc (Carignan \& Purton 1998). Nevertheless, the solid and dashed lines achieve excellent fits up

Table 1. Here we show the various parameters corresponding to our fitted models that are plotted in Figs 6-10). For each galaxy we give the measured parameters from the literature and our fitted values. The measured distances come from Walter et al. (2008), who took their value from Freedman et al. (2001), except NGC 2403 which comes from the more recent study of Vinkó et al. (2006). The masses for the stellar and gaseous components come from de Blok et al. (2008) and are correct at the mean of the measured distance and with use of the diet-Salpeter initial mass function. The predicted mass-to-light ratios ( $M / L$ values) also come from de Blok et al. (2008), for which 'd-S' and ' $\mathrm{Kr}$ ' correspond to diet-Salpeter and Kroupa initial mass functions, respectively. The suggested stellar scale-heights are merely one-fifth of the radial scale-height. There is no suggested scale-height for the gaseous distribution. For each galaxy, every separate fit is given a different letter $-\mathrm{a}, \mathrm{b}, \mathrm{c}$ or $\mathrm{d}-$ and these fits correspond to different line types in their corresponding figures. Our fitted distances and values for the $M / L$ are normalized to the mean measured distance and the diet-Salpeter IMF, respectively. The stellar scale-heights are well constrained, but the gas scale-heights less so. For DDO 154 we fixed the stellar parameters since they had little influence.

\begin{tabular}{|c|c|c|c|c|c|c|c|c|}
\hline Galaxy & $\begin{array}{l}\text { Measured distance } \\
(\mathrm{Mpc})\end{array}$ & $\frac{\text { Fitted distance }}{\text { Measured distance }}$ & $\begin{array}{l}\text { Stellar (gas) mass } \\
\qquad\left(10^{9} \mathrm{M}_{\odot}\right)\end{array}$ & $\begin{array}{c}\text { Predicted } M / L \\
{[3.6 \mu \mathrm{m} \mathrm{d}-\mathrm{S}(\mathrm{Kr})]}\end{array}$ & $\frac{\text { Fitted } M / L}{\mathrm{dS} M / L}$ & $\begin{array}{l}\text { Suggested } z_{*} \\
\quad(\mathrm{kpc})\end{array}$ & $\begin{array}{l}\text { Fitted } z_{*}\left(z_{\mathrm{g}}\right) \\
\qquad(\mathrm{kpc})\end{array}$ & Line type \\
\hline DDO 154-a & \multirow[t]{2}{*}{$4.30 \pm 1.07$} & 1.085 & \multirow[t]{2}{*}{$0.026(0.468)$} & \multirow[t]{2}{*}{$0.32(0.23)$} & 1.0 & \multirow[t]{2}{*}{0.2} & $0.2(1.5)$ & Solid \\
\hline DDO 154-c & & 0.9 & & & 1.0 & & $0.2(0.05)$ & Dotted \\
\hline NGC 3621-a & \multirow[t]{2}{*}{$6.64 \pm 0.70$} & 0.91 & \multirow[t]{2}{*}{$19.3(9.58)$} & \multirow[t]{2}{*}{$0.59(0.42)$} & 0.88 & \multirow[t]{2}{*}{1.5} & $0.5(1.0)$ & Solid \\
\hline NGC 3621-b & & 0.97 & & & 0.76 & & $0.5(1.0)$ & Dashed \\
\hline NGC 2903-a & \multirow[t]{3}{*}{$8.90 \pm 2.20$} & 0.8 & \multirow[t]{3}{*}{$16.2(6.6)$} & \multirow[t]{3}{*}{$0.61(0.43)$} & 4.6 & \multirow[t]{3}{*}{0.5} & $0.54(1.0)$ & Solid \\
\hline NGC 2903-b & & 1.1 & & & 2.5 & & $0.3(1.0)$ & Dashed \\
\hline NGC 2903-c & & 1.15 & & & 1.5 & & $0.2(1.0)$ & Dotted \\
\hline NGC 2403-a & \multirow[t]{2}{*}{$3.47 \pm 0.29$} & 1.19 & \multirow[t]{2}{*}{$5.13(3.82)$} & \multirow[t]{2}{*}{$0.39(0.26)$} & 1.15 & \multirow[t]{2}{*}{0.4} & $0.4(0.1)$ & Solid \\
\hline NGC 2403-b & & 1.09 & & & 1.45 & & $0.5(0.1)$ & Dashed \\
\hline
\end{tabular}



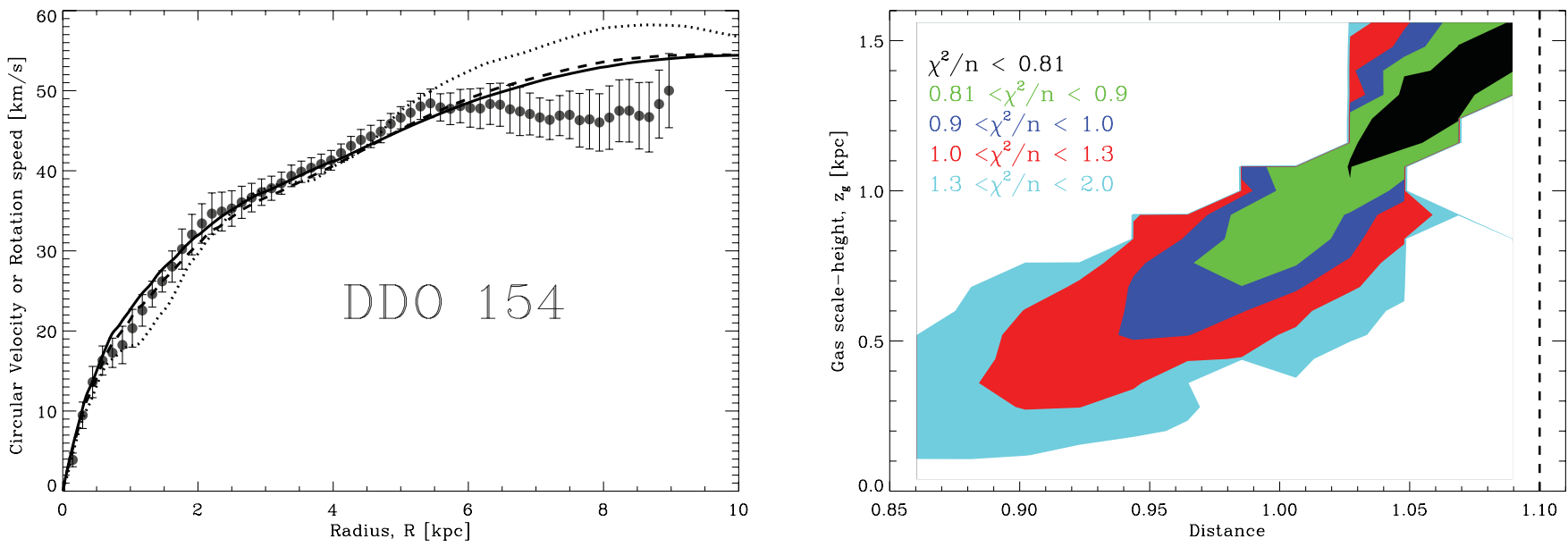

Figure 6. In the left-hand panel we plot the rotation curve of DDO 154 as measured by de Blok et al. (2008) along with our MOND fits. The curves are described in Table 1, giving their line types and model parameters. The right-hand panel shows contours of reduced $\chi^{2}$ for the two free parameters: the distance normalized by the mean measured distance and the gas scale-height. The contour levels are defined in the panel and the vertical dashed line defines the $1 \sigma$ error on the measured distance.
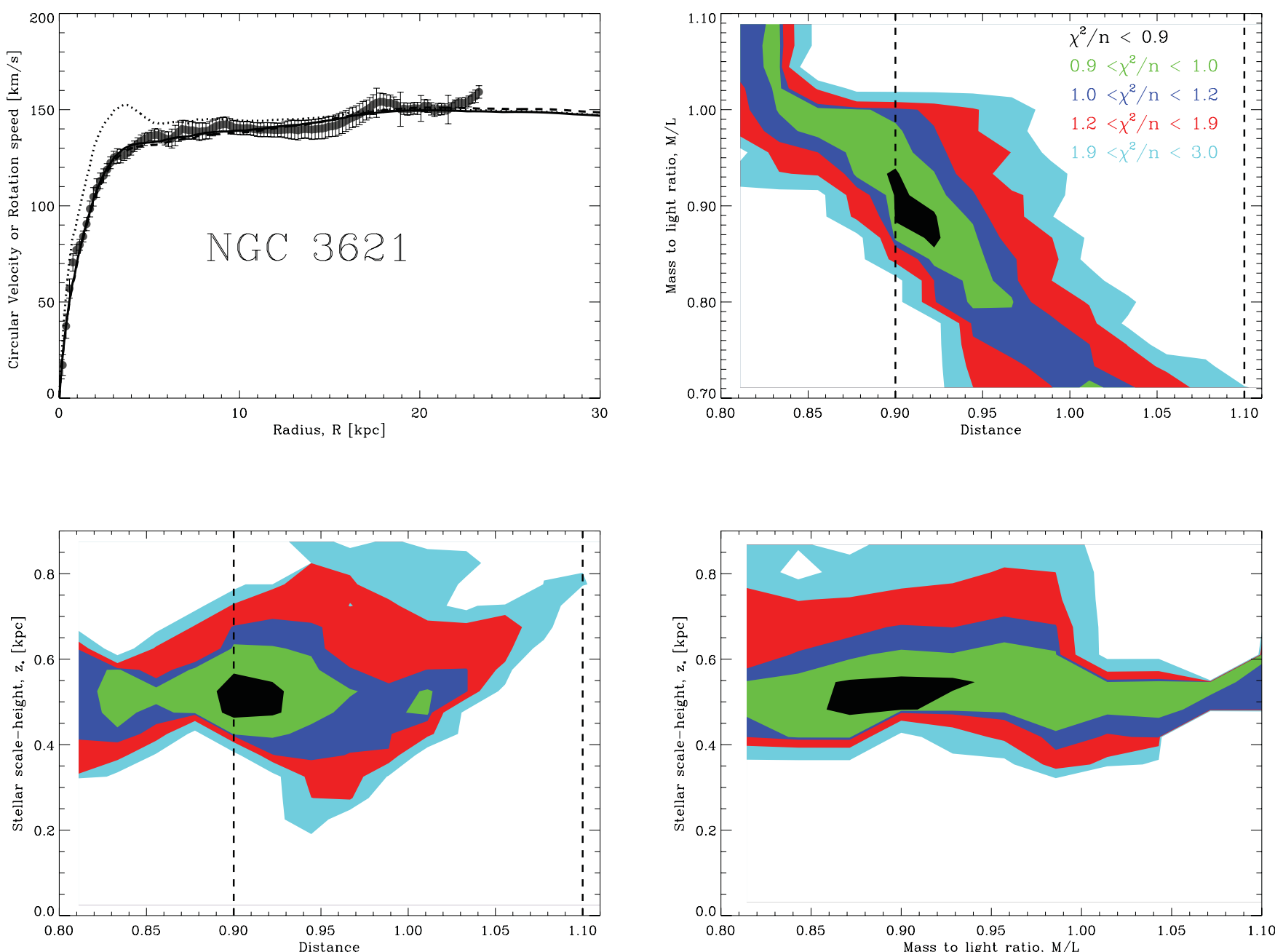

Figure 7. In the top left-hand panel we plot the rotation curve of NGC 3621 as measured by de Blok et al. (2008) along with our MOND fits. The curves are described in Table 1, giving their line types and model parameters. The other three panels show contours of reduced $\chi^{2}$ for the three combinations of three free parameters; these are the fitted distance and $M / L$ normalized to the measured distance and diet-Salpeter IMF, respectively, and the fitted stellar scale-height. The contour levels are defined in the top right-hand panel. The vertical dashed line in the contour plots with distance defines the $1 \sigma$ error on the measured distance. 

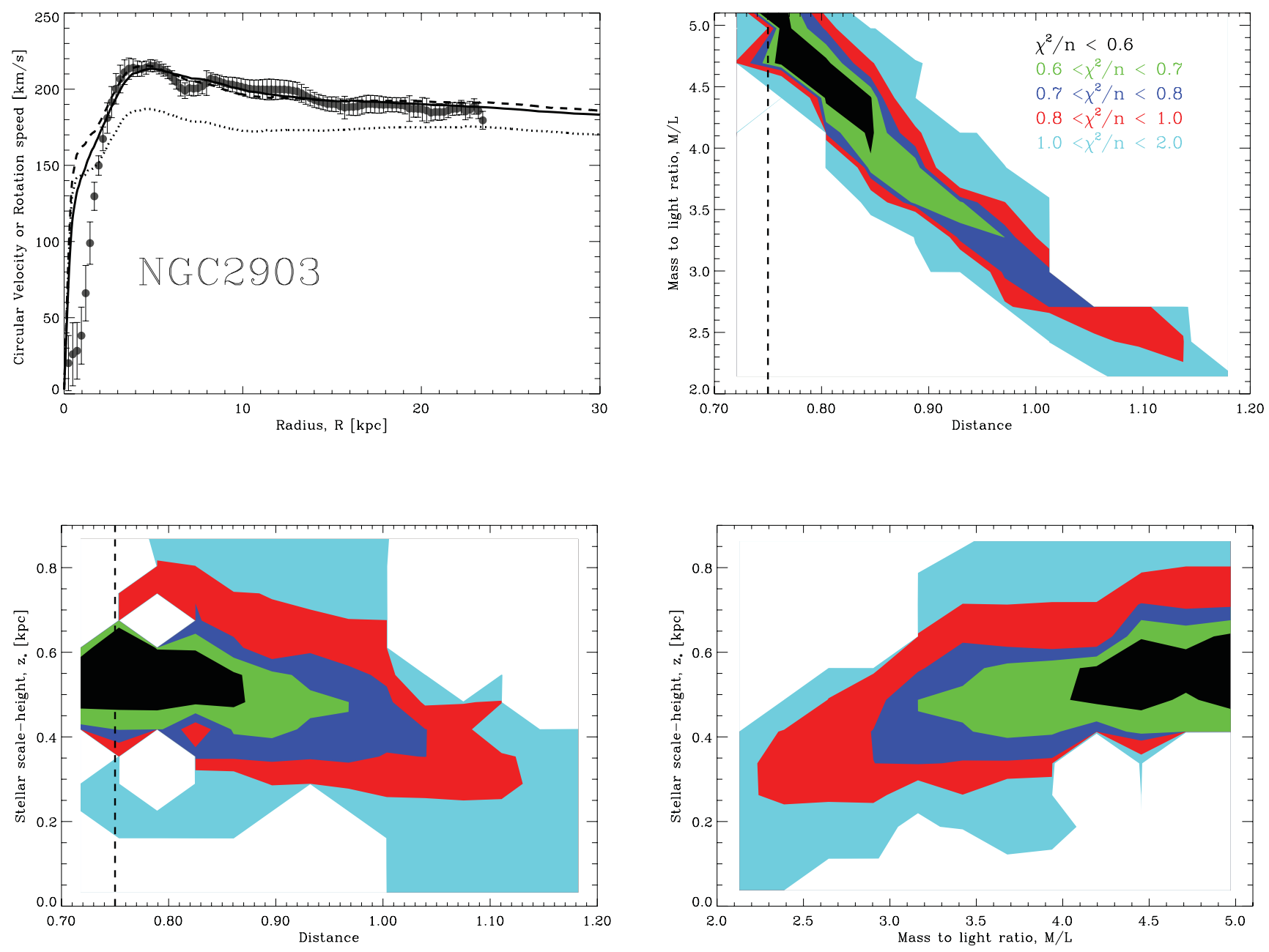

Figure 8. Same as Fig. 7, but for NGC 2903.

to $6 \mathrm{kpc}$ and the dashed line uses an acceptable distance and gas scale-height of only $0.65 \mathrm{kpc}$. The dotted line uses a razor thin gas disc and is a poor fit to the inner curve and poorer still to the outer curve. Walter \& Brinks (1999) claim that dwarf galaxies (such as DDO 154) have thicker $\mathrm{H}$ I discs than more massive spiral galaxies due to a lower restoring force from weaker gravity. In order to be a good fit in MOND, the outer warp should be responsible for the anomalously low rotation speeds between 7 and $8.5 \mathrm{kpc}$.

\subsection{NGC 3621}

The $\chi^{2}$ minimum for NGC 3621 is obvious in the contour plots of Fig. 7 and puts strong constraints on all three parameters. The fits, whose parameters are given in Table 1, at the top left-hand panel show the best fit (solid line) and another fit with slightly larger distance (dashed line), more in keeping with the measured distance. Both are excellent fits. The dotted line shows the best-fitting model when the best-fitting stellar scale-height is replaced with a razor thin stellar disc and is a very poor fit to the central rotation curve. The best-fitting parameters are all within acceptable bounds and this is an excellent fit for MOND.

\subsection{NGC 2903}

The contour plots in Fig. 8 show that in order to have a satisfactory fit, regardless of distance and stellar scale-height, the $M / L$ must be more than twice the predicted value. If this is the case, then an excellent fit can be achieved as shown in the top left-hand panel. On the other hand, using a $M / L$ of only 50 per cent larger than the predicted value gives a poor fit, as the dotted line attests. Therefore, NGC 2903 is a problem for MOND unless a reason can be found why it should have a $M / L$ that is twice the diet-Salpeter prediction. If such a reason was found, it would be well fitted by MOND.

\subsection{NGC 2403}

In Fig. 9 the contour plot of distance against $M / L$ shows the requirement for a good fit to have either an excessively large $M / L$ or distance. This is aggravated by the tight error on the distance of a mere 8 per cent and the relatively low predicted $M / L$ for the given stellar population. For instance, the predicted $3.6 \mu \mathrm{m}$ diet-Salpeter IMF $M / L$ of NGC 2403 is 0.39 , whereas for NGC 2903, NGC 3521 and NGC 3621 it is $0.61,0.73$ and 0.59 , respectively.

In the top left-hand panel of Fig. 9 we fit four curves to the observed rotation curve. The solid and dashed curves are the bestfitting models, which, respectively, use a $>2 \sigma$ discrepant fitted distance and regular $M / L$, and a more regular distance but more discrepant $M / L$. The other two lines are a model with the same parameters as the best fit, but using a razor thin stellar disc (dotted line), and one with a regular distance and $M / L$ (dot-dashed line). 

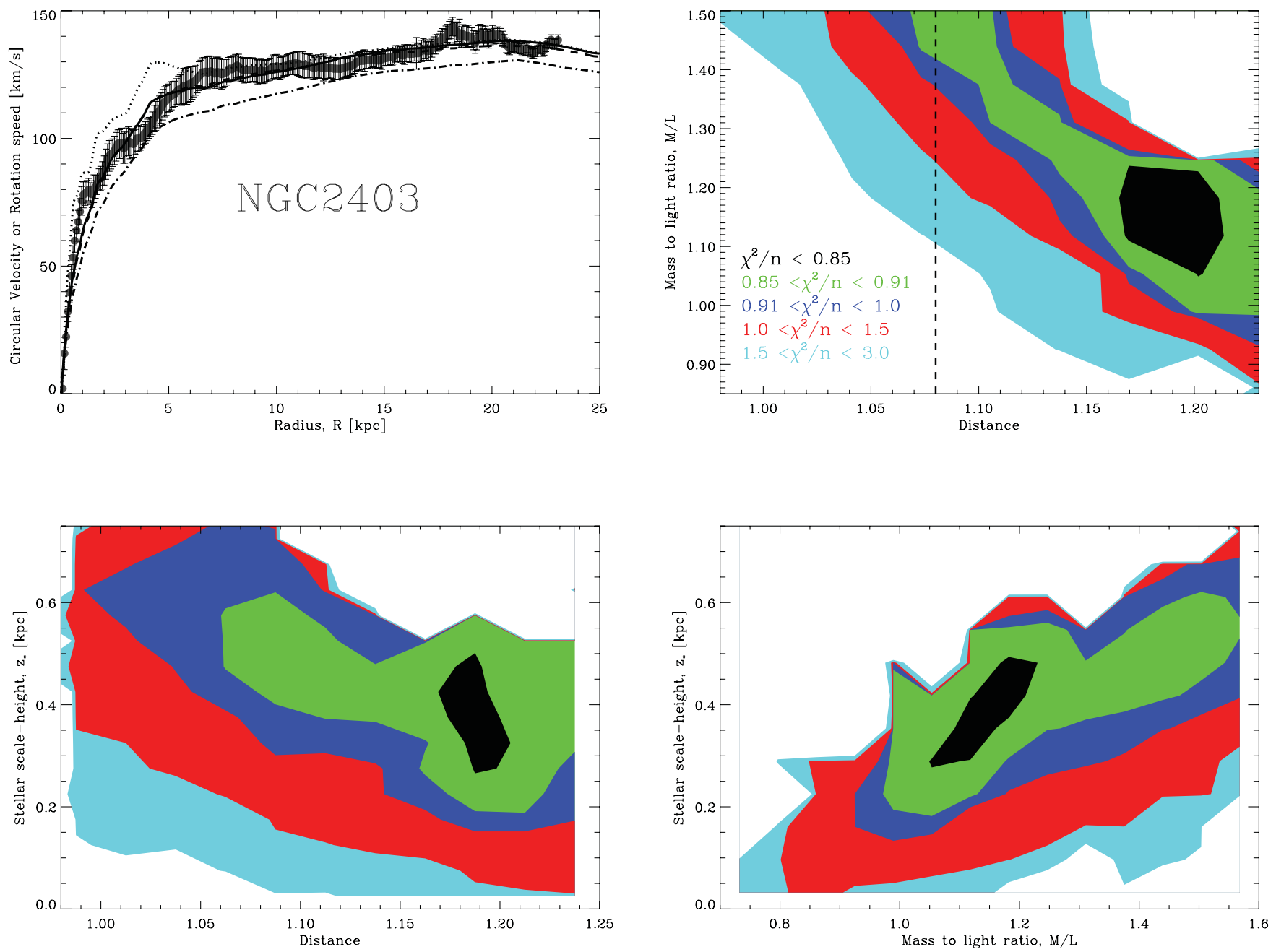

Figure 9. Same as Fig. 7, but for NGC 2403.

Both of these are unsatisfactory fits. As with NGC 2903, this galaxy NGC 2403 can only be consistent with MOND if the cause of its higher than expected $M / L$ is found; however, this is a much less significant problem than NGC 2903 because even with the required $M / L$ for a good fit, it would still have a typical $M / L$ compared to other galaxies.

\subsection{NGC 3521}

For NGC 3521, the preference is for a much lower distance than measured, which can be seen in the contour plot of Fig. 10. This is not necessarily out of the question since the error on its distance is 30 per cent, but the $\chi^{2}$ minimum is actually further from the mean than this. The parameters of the two models plotted against the observed rotation curve in the top left-hand panel are given in Table 1 and correspond to a distance more than $1 \sigma$ lower than the mean and roughly $0.5 \sigma$ lower than the mean. Both require sensible $M / L$ and the stellar scale-height is constrained to be close to $0.25 \mathrm{kpc}$. The fit with the less discrepant distance (dashed line) is a better fit to the inner $13 \mathrm{kpc}$ and the discontinuity in measured rotation speed at this radius makes it difficult for any model to achieve a good fit to both the inner and outer curves. In general, this galaxy is well fitted by MOND.

\subsection{Stellar scale-heights}

A somewhat surprising result is the tight constraint placed on the values of the stellar scale-height for the four stellar dominated galaxies and the gas scale-height of DDO 154. From the contour plots of Figs 6-10 we see the relative lack of freedom in the stellar scaleheights and this gives us the opportunity to put a further constraint on the model by checking if the required scale-heights are consistent with the typical stellar vertical velocity dispersions of other spiral galaxies.

Making the assumption that the stellar velocity dispersions are isothermal with height above the disc we can use the following equation to solve for the stellar vertical velocity dispersion at any radius:

$\sigma_{z, *}^{2}(R)=-\frac{\mathrm{d} \Phi(R, z)}{\mathrm{d} z} \times \frac{\rho_{*}(R, z)}{\partial_{z} \rho_{*}(R, z)}$.

A similar test could be used to constrain the gas scale-heights, but we do not follow that route. The right-hand side is comprised fully of quantities that are known in the rotation curve fitting code. As can be seen in Fig. 11, the vertical velocity dispersions of all the galaxies have a similar shape. Generally, for the best-fitting models (solid and dashed lines) the trend is to have a large central vertical velocity dispersion $\sigma_{z}(0)$ of between 20 and $35 \mathrm{~km} \mathrm{~s}^{-1}$ and this drops to between 4 and $8 \mathrm{~km} \mathrm{~s}^{-1}$ by $15 \mathrm{kpc}$. The dotted lines, which 

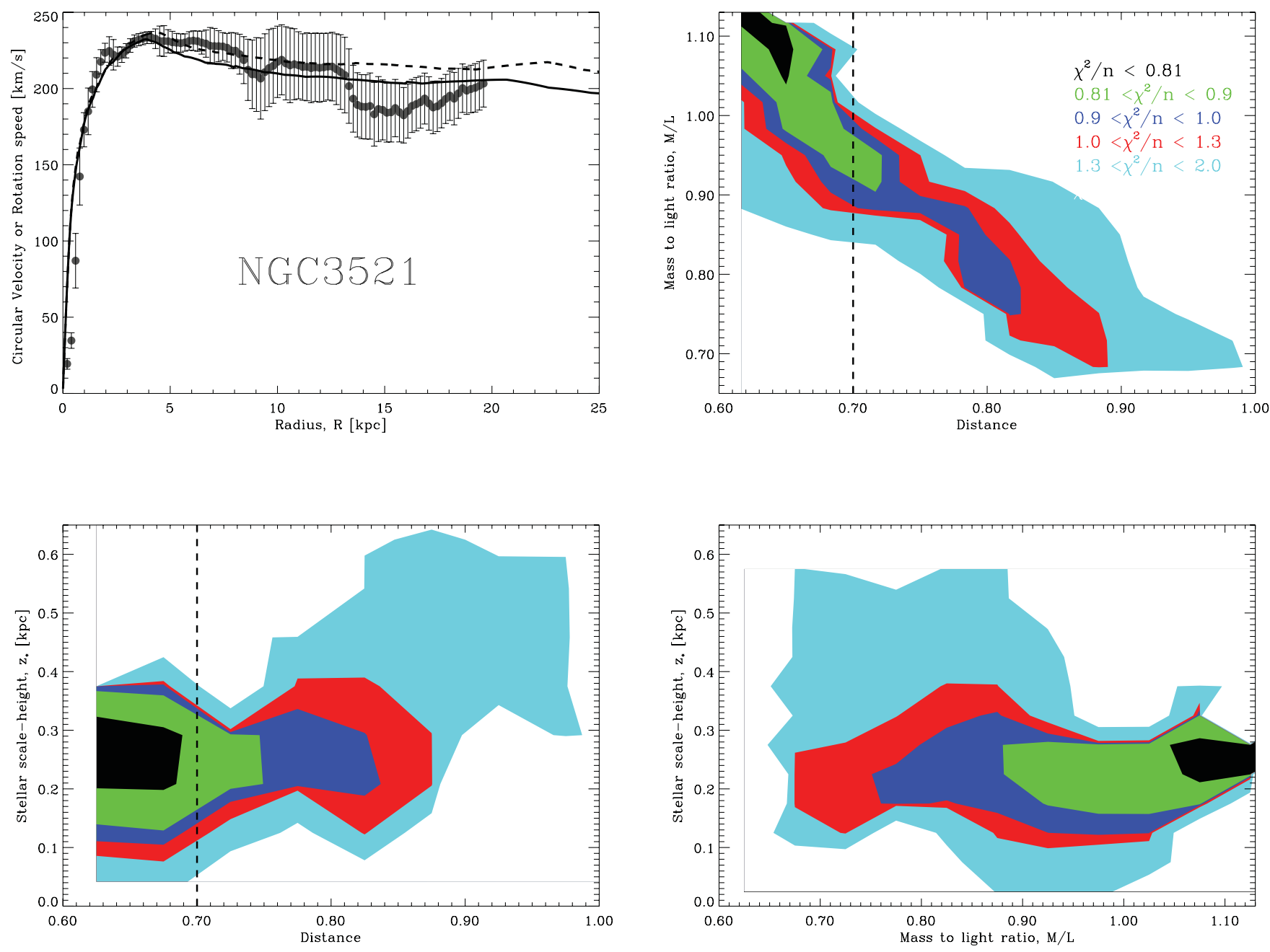

Figure 10. Same as Fig. 7, but for NGC 3521.

are for very thin discs of only $0.05 \mathrm{kpc}$ scale-height, have much lower velocity dispersions - with NGC 2403 and NGC 3621 being centrally just 8 and $10 \mathrm{~km} \mathrm{~s}^{-1}$, respectively.

Bottema (1993) studied the stellar velocity dispersions of a sample of 12 spiral galaxies of varying blue band magnitude in the range $M_{B}=-18.76$ to -22.22 . They found a trend for the vertical stellar velocity dispersions to scale with decreasing galaxy magnitude (increasing luminosity). The three galaxies with $M_{B}>-20$, for which only NGC 3521 from our sample does not apply, were NGC 3198, NGC 3938 and NGC 6503 and they had central stellar velocity dispersions of $45 \pm 5,32 \pm 13$ and $30 \pm 7 \mathrm{~km} \mathrm{~s}^{-1}$, respectively, although NGC 3198 and NGC 3938 are inclined. The stellar velocity dispersions dropped to between 10 and $20 \mathrm{~km} \mathrm{~s}^{-1}$ after 2 or 3 scale-heights, which is similar to our models. The larger galaxies with $M_{B}<-21$, which applies to NGC 3521, had central stellar velocity dispersions between 50 and $120 \mathrm{~km} \mathrm{~s}^{-1}$. This is larger than the central stellar velocity dispersion of NGC 3521. Even increasing the stellar scale-height to $1 \mathrm{kpc}$ can only increase the central vertical stellar velocity dispersion to $60 \mathrm{~km} \mathrm{~s}^{-1}$ and this remains on the low end of the sample studied by Bottema (1993). Perhaps the increased velocity dispersions with luminosity are also linked to the increased prevalence of bulges with luminosity and NGC 3521's lack of a significant spheroid bucks this trend.
The stellar and gas scale-heights we use in the fits are given in Table 1 along with the stellar scale-heights suggested by de Blok et al. (2008). The suggested stellar scale-heights are simply one-fifth of the scale-lengths and for NGC 3521 and NGC 3621, respectively, are 1.9 and $1.5 \mathrm{kpc}$. These values are several times larger than the fitted scale-heights of 0.25 and $0.5 \mathrm{kpc}$, respectively. If these suggested scale-heights were enforced, it would not be possible to achieve a good fit to the rotation curves. Therefore, it is vital to have a separate measurement of the stellar velocity dispersion to put an orthogonal limit on the scale-heights, as shown by Puglielli, Widrow \& Courteau (2010) in the case of NGC 6503 and Bershady et al. (2011). Bershady et al. (2011) used the Disk Mass Survey to demonstrate that the magnitude of vertical velocity dispersions led to the inference that galaxy discs must be submaximal. Maximum disc simply means the fit to the rotation curve with the highest $M / L$ possible and the least amount of dark matter, such that all the rotation velocity is attributable to the disc in the central part. It would be interesting to see if this holds in MOND for which the disc, by definition, must be maximal.

Banerjee et al. (2011) made an analysis of the scale-heights of the gas-dominated THINGS galaxies in Newtonian gravity and it is essential that a similar review is made of the same galaxies in MOND. 

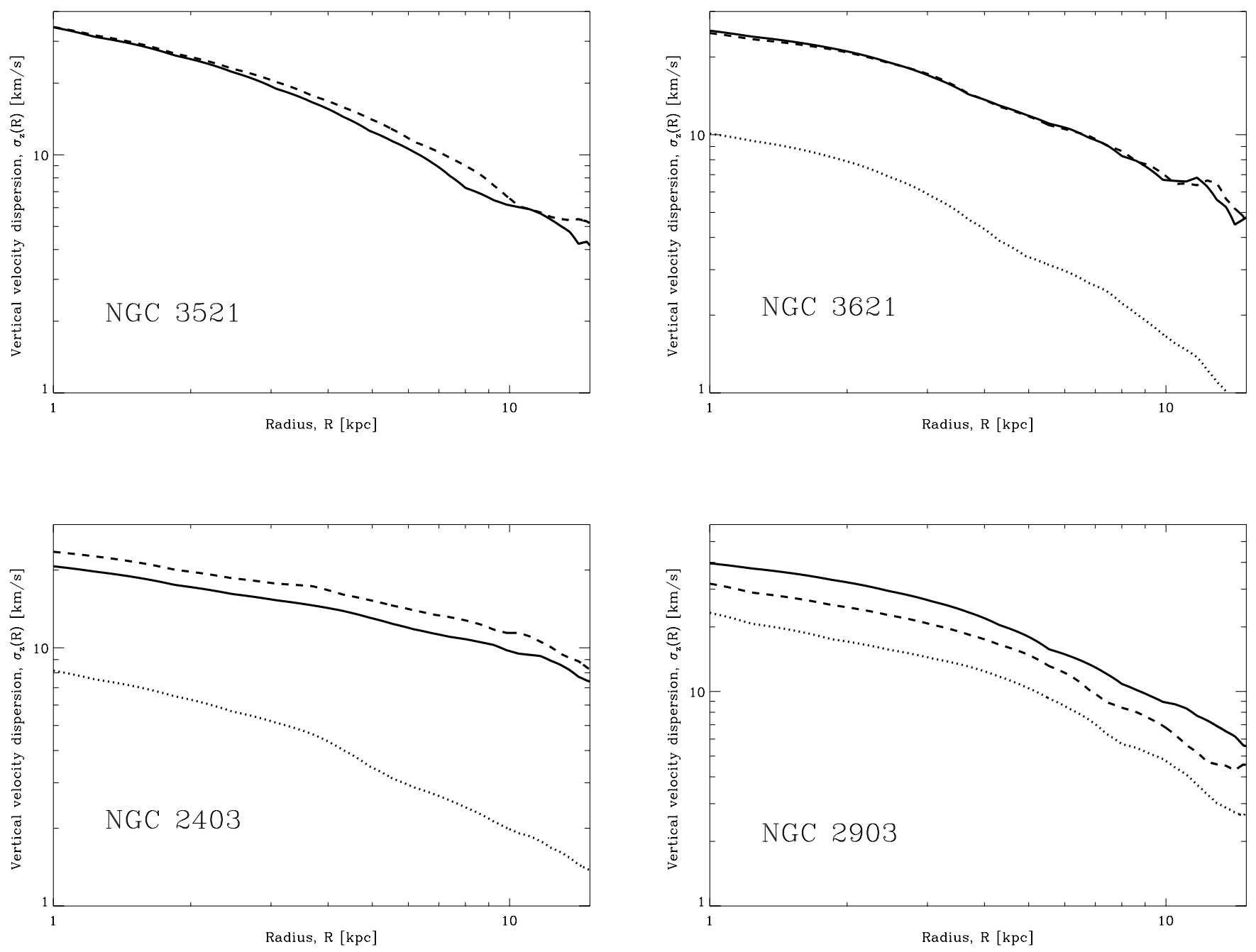

Figure 11. Stellar vertical velocity dispersions as functions of radius for the four stellar dominated galaxies. The line types for each galaxy are defined such that the solid, dotted and dashed lines always correspond to models (a), (b) and (c), respectively, as set out in Table 1.

\subsection{The external field effect}

External gravitational fields (see forthcoming paper), usually the result of nearby galaxies or clusters, can cause a suppression of the boost to gravity due to MOND. This is particularly likely for low surface brightness galaxies. An external gravitational field has a similar effect to decreasing the $M / L$ and increasing the scaleheight, but they are not degenerate given the gaseous mass content and the radial dependence of the circular velocity on scale-height. It is an oft-quoted solution to unknown problems in MOND, but it is important to emphasize that it would have no beneficial influence on NGC 2403 or NGC 2903 since it would simply impose a larger $M / L$, making the situation worse.

\section{CONCLUSION}

Here we have introduced an $N$-body code that solves the modified Poisson equation of QUMOND (see Milgrom 2010). With it, we fitted the rotation curves of five spiral galaxies from the THINGS survey (Walter et al. 2008), using $N$-body realizations of the stars and the gas in each galaxy fixed by their surface densities. We allowed the distance, $M / L$ and both the scale-heights of the stellar and gaseous discs to be free parameters, with priors set depending on observational constraints.
We discovered that our best fits were excellent matches to the rotation curves of all five galaxies, except for some minor discrepancies at locations of publicized uncertainty in the observations. We displayed contour plots of reduced $\chi^{2}$ for the various free parameters of all galaxies and found that two galaxies, NGC 2403 and NGC 2903, could only make satisfactory fits if their $M / L$ values were larger than the predicted values. The required increase in $M / L$ for NGC 2403 is moderate, but for NGC 2903 it is considerable. Interestingly, the dark matter fits to these rotation curves given by de Blok et al. (2008), which can be seen in their tables 5 and 6 , also require larger $M / L$. For NGC 2403 , a value 50 per cent larger than the predicted diet-Salpeter value and for NGC 2903 more than double was required, which is exactly what we have found.

If this were an isolated incident, it could have less relevance, but there is a growing preference for stellar dominated galaxies to require much larger $M / L$ values than predicted by stellar population models from typical initial stellar mass functions. Large $M / L$ values are also required for certain dwarf spheroidal galaxies surrounding the Milky Way, in particular Carina, Sextans and Draco (see Angus 2008; Serra, Angus \& Diaferio 2010), and a large fraction of the early-type galaxies studied by Sanders \& Noordermeer (2007). The MOND fits to the dynamics are in general still excellent, but a solution must be found to explain why certain galaxies can have 
$M / L$ described by a Kroupa or diet-Salpeter IMF and other galaxies need one up to twice as large. Both the MOND and dark matter fits to the rotation curves of NGC 2403 and NGC 2903 suggest that there is a problem with the stellar population synthesis models of these galaxies. Furthermore, the distance required for NGC 3521 is considerably lower than the mean, but this is acceptable due to the large uncertainty. A revised distance with tighter error bars could be very revealing. These issues need to be resolved before we can say that MOND provides good fits to all five galaxies with reasonable parameters.

The surprising result was that the MOND fits put a tight constraint on the stellar scale-heights. The best-fitting scale-heights of all four stellar dominated galaxies were found to be between 0.25 and $0.55 \mathrm{kpc}$ and were strongly constrained to be larger than 0 and less than $1 \mathrm{kpc}$. For two galaxies, NGC 2403 and NGC 2903, the best-fitting stellar scale-heights were very close to the typical scaleheight used, which is one-fifth of the radial scale-length. On the other hand, NGC 3521 and NGC 3621 have best-fitting stellar scaleheights that are far lower than the suggested scale-height insofar as if the scale-height was fixed to this suggested value, a good fit to the inner data points would not be achievable. Similarly, the only gas-dominated galaxy we studied, DDO 154, can achieve a good fit only if its gas scale-height is larger than $\sim 0.6 \mathrm{kpc}$. In fact, the quality of the fit improves with increasing scale-height.

Clearly, the use of both stellar and gas scale-heights as free parameters in MOND fits to galaxy rotation curves is imperative. Line-of-sight velocity dispersions of both components in a sample of similar face-on galaxies should be used as a sanity check on their values since in MOND the rotation curves and vertical velocity dispersions of both components are bound together to the mass distribution.

\section{ACKNOWLEDGMENTS}

GWA's research is supported by the Claude Leon Foundation and a University Research Fellowship. GWA, KvdH and WJGdB's research is supported by the University of Cape Town and the National Research Foundation of South Africa. BF acknowledges the support of the AvH Foundation. GG is a postdoctoral fellow with the National Science Fund (FWO-Vlaanderen). The authors thank the THINGS collaboration for providing their data.

\section{REFERENCES}

Angus G. W., 2008, MNRAS, 387, 1481

Angus G. W., Diaferio A., 2011, MNRAS, 417, 941
Banerjee A., Jog C. J., Brinks E., Bagetakos I., 2011, MNRAS, 415, 687

Bekenstein J., Milgrom M., 1984, ApJ, 286, 7

Bershady M. A., Martinsson T. P. K., Verheijen M. A. W., Westfall K. B., Andersen D. R., Swaters R. A., 2011, ApJ, 739, L47

Binney J., Tremaine S., 2008, Galactic Dynamics, 2nd edn. Princeton Univ. Press, Princeton, NJ

Bottema R., 1993, A\&A, 275, 16

Brada R., Milgrom M., 1995, MNRAS, 276, 453

Brada R., Milgrom M., 1999, ApJ, 519, 590

Brada R., Milgrom M., 2000a, ApJ, 541, 556

Brada R., Milgrom M., 2000b, ApJ, 531, L21

Carignan C., Purton C., 1998, ApJ, 506, 125

de Blok W. J. G., Walter F., Brinks E., Trachternach C., Oh S., Kennicutt R. C., 2008, AJ, 136, 2648

Famaey B., Binney J., 2005, MNRAS, 363, 603

Famaey B., McGaugh S., 2011, preprint (arXiv:1112.3960)

Freedman W. L. et al., 2001, ApJ, 553, 47

Gentile G., Famaey B., de Blok W. J. G., 2011, A\&A, 527, A76

Kennicutt R. C., Jr, et al., 2003, PASP, 115, 928

Llinares C., 2011, PhD thesis, Kapteyn Astronomical Institute, Groningen

Llinares C., Knebe A., Zhao H., 2008, MNRAS, 391, 1778

Londrillo P., Nipoti C., 2009, Mem. Soc. Astron. Ital., 13, 89

Milgrom M., 1983, ApJ, 270, 365

Milgrom M., 1986, ApJ, 302, 617

Milgrom M., 2010, MNRAS, 403, 886

Nipoti C., Londrillo P., Ciotti L., 2007a, ApJ, 660, 256

Nipoti C., Londrillo P., Ciotti L., 2007b, MNRAS, 381, L104

Nipoti C., Ciotti L., Binney J., Londrillo P., 2008, MNRAS, 386, 2194

Press W. H., Teukolsky S. A., Vetterling W. T., Flannery B. P., 1992, Numerical Recipes in fortran. The Art of Scientific Computing. Cambridge Univ. Press, Cambridge

Puglielli D., Widrow L. M., Courteau S., 2010, ApJ, 715, 1152

Sanders R. H., Noordermeer E., 2007, MNRAS, 379, 702

Serra A. L., Angus G. W., Diaferio A., 2010, A\&A, 524, 16

Tiret O., Combes F., 2007, A\&A, 464, 517

Tiret O., Combes F., 2008a, A\&A, 483, 719

Tiret O., Combes F., 2008b, in Funes J. G., Corsini E. M., eds, ASP Conf. Ser. Vol. 396. Formation and evolution of galaxy disks. Astron. Soc. Pac., San Francisco, p. 259

Vinkó J. et al., 2006, MNRAS, 369, 1780

Walter F., Brinks E., 1999, AJ, 118, 273

Walter F., Brinks E., de Blok W. J. G., Bigiel F., Kennicutt R. C., Thornley M. D., Leroy A., 2008, AJ, 136, 2563

Wu X., Zhao H., Wang Y., Llinares C., Knebe A., 2009, MNRAS, 396, 109

Zhao H., Famaey B., 2010, Phys. Rev. D, 81, 087304

This paper has been typeset from a $\mathrm{T}_{\mathrm{E}} \mathrm{X} / \mathrm{LAT} \mathrm{E} \mathrm{X}$ file prepared by the author. 